\title{
Social Media Application for Specially Challenged
}

This paper was downloaded from TechRxiv (https://www.techrxiv.org).

\section{LICENSE}

CC BY 4.0

SUBMISSION DATE / POSTED DATE

$22-12-2021 / 27-12-2021$

CITATION

J, Nishchal (2021): Social Media Application for Specially Challenged. TechRxiv. Preprint. https://doi.org/10.36227/techrxiv.17371748.v1

$\mathrm{DOI}$

10.36227/techrxiv.17371748.v1 


\section{Social Media Application for Specially Challenged}

\author{
Nishchal J \\ $R V$ College of Engineering \\ nishchalj.cs18@rvce.edu.in
}

\author{
Navya Priya $N$ \\ $R V$ College of Engineering \\ navyapriyan.cs18@rvce.edu.in
}

\author{
Pratiba D \\ $R V$ College of Engineering \\ pratibad@rvce.edu.in
}

\begin{abstract}
Every person has an equal right to information, therefore, impairments shouldn't restrict people from gaining this knowledge from any form of source. Social Networking applications have tremendously grown their popularity among all kinds of age groups for providing socialising opportunities, entertainment and exchange of knowledge. Hence, the motive of this paper is to propose a social networking application pipeline with a strong Machine Learning backend which makes it more accessible to the blind, deaf and dumb section of the society who otherwise do not enjoy the features of social networking platforms.
\end{abstract}

Keywords- Indian Sign language(ISL), Image Captioning, Speech recognition, Text-to-Speech, Optical Character Recognition(OCR), GIF Captioning, Long Short Term Memory(LSTM), Natural language processing(NLP), Convolutional neural networks(CNN).

\section{INTRODUCTION}

Social networking can have various purposes, be it social, business, or both, through sites like Facebook, Twitter, LinkedIn, and Instagram. Social networking has become a prominent base for companies seeking to engage customers. Regardless of some stiff competition, Facebook remains the biggest and most well known social networking site, with about 2.8 billion active users every month, as of Dec. 31, 2020. The others included Instagram, Facebook Messenger, Twitter, and Pinterest, in order of popularity, according to Statistica.

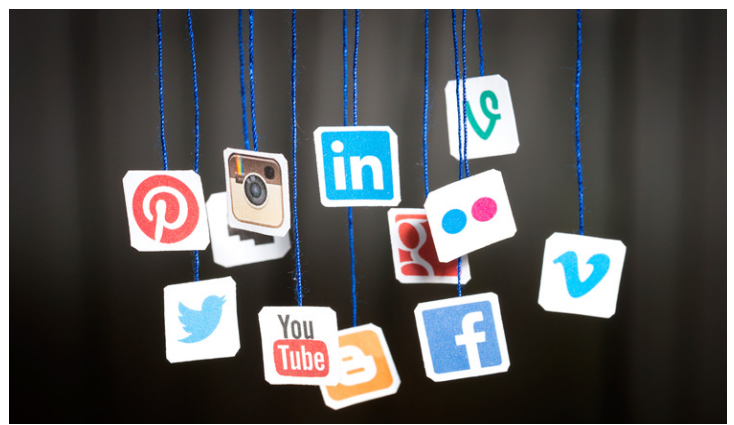

FIG 1. Various social media platforms and their influence in the world.

However, technological advancements cause a great deal of confusion among people who use access technologies, when it comes to accessing these social media networks.This confusion might be attributed to social networks being rather intimidating to those who have visual impairments and have yet to access them. Subsequently, it's fairly simple to overcome this confusion with a solution along the lines of not really wanting to be engaged with social networks or being too busy to even take part.
Actually,social networks are a boon, which have great deals to offer to the world.Once the idea behind it is firmly grasped and access obstacles dealt with, social networking for people who are specially challenged can be rewarding, fulfilling, and fun[9][12]. The same problem is faced by the deaf and dumb people who cannot enjoy the amu audio experience of videos posted on social networking applications[13]. Not all videos can have transcripts provided instantly. Moreover, there can be people who might be well versed with sign languages only, hence English transcripts can be of little use to them.

Hence it is noticed that, though the Blind people can enjoy the visual experience of the Social Media Applications, they cannot utilise any visual data present, on the other hand, the deaf and dumb can experience memes and GIF's and Image posts however, they cannot enjoy the audial entertainment provided by networking sites. Therefore, there is a need for creation of a pipeline which can accommodate certain extra features like Speech to Text,Text to Speech, Speech to Sign Language along with Image/GIF/Meme captioning, so that none of the sections in the society feel left out, and every person can exercise their right to information and entertainment[6].

\section{Literature SURVEY}

Social media is globally recognised for bringing great value to the users. By associating people and propagating information among them, social media fosters communities, spreads new opinions and behaviors, conveys critical information rapidly in reaction to crises, and even leads to cultural impact. Various web applications have been created just for the specially challenged, but these applications do not cater to all of them that is blind, deaf and dumb together. Numerous past studies also looked at how different social groups engage with on-line social networks. There has been an extensive study carried out on how visually challenged people dwell with social networking platforms, specifically Facebook. A sample of 50k visually challenged subjects were used to their browning activities, social networking tendencies they built on Facebook. So, the analysis was done only for the visually impaired and not for the deaf or dumb. Also, the existing Facebook application was being used which has no special provisions for the deaf and dumb.[2] Hence, the work done by Shaomei $\mathrm{Wu}$ and Lada Adamic had minimal but useful implications for improving the usability and utility of online social networks for visually impaired users.

There is also a survey done by Nora Griffin-Shirley, Devender R. Banda, Paul M. Ajuwon, Jongpil Cheon, Jaehoon Lee, Hye Ran Park, and Sanpalei N. Lyngdoh where they explored the usage as well as their perceptions of mobile phones and various social networking apps among visually challenged people. The survey dealt with sociodemographic information such as visual functioning, gender, age, ethnicity, geographical 
location, country, occupation, education, and annual income and the use of mobile devices and apps in general which consisted of years of using a mobile device, device type, apps in use, the number of apps downloaded in a month, frequently used apps, usability, accessibility of apps, and use of apps specifically designed for people who are visually challenged. Although the survey lacked diversity in geographical location in spite of their claim, it was found out that more than $90 \%$ of the visually impaired who took part in the survey conveyed that they were currently using apps that were especially designed for people with visual impairments. Hence, it shows that specially challenged people tend to use apps that are designed specially for them keeping in mind the difficulties they face.[3]

A survey was conducted by Mrim Alnfiai, Srini Sampali which studied the existing features present in communication apps which are found useful by the deaf and dumb. They move ahead and also suggest some useful features which can be possibly implemented on the apps in the future, draw a comparison of them with existing ones and prove their necessity in future. The features in the application like size of buttons,sign language, support for several languages, notifications, joining social media, text to speech, speech to text are the desirable features and the frequency of these in existing apps is quite less. All these barriers limit the usage of social media apps[4].

The principal impact of social media on the way disabled people integrate to modern society was also studied by Masuma H. Mammadova, Sanan M. Ahmadov where the needs and purposes of disabled people can be met through social media were analysed along with additional functionalities designed especially for disabled people. This paper discussed the various problems that impaired people face while using social media apps and also showed ways to combat them. It is for sure a known fact that the Government plays a vital role in stimulating, promoting and developing applications which are economically produced and affordably sold to the target consumers. Hence, the government should join hands with the tech companies to promote the introduction of special features. In conclusion, though many social networking apps like Instagram or facebook do try to accommodate the physically challenged section of society with some integration efforts, unfortunately they have not achieved the full resolution of the problem.[5].

A survey was conducted to document the social media use among people with visual impairments in Norway, and to explore some barriers and motivational factors to the use of social media for this group.In conclusion of the survey it was found that reliance on guidelines and National legislation does not ensure accessibility, and the challenges cannot be solved by better accessibility guidelines alone.A user centered and participatory development process, which involves various stakeholders, is needed. These aspects should be highlighted when enforcing universal design as a legal concept. Hence, we have tried to involve various stakeholders in order to take into consideration all of their insights[1].

Here, in this paper we have made an attempt to include all the necessary features so that the application is user friendly for everyone and also one application that caters to blind,deaf and dumb.

\section{Proposed Methodology}

The proposed application pipeline has the following features embedded to a normal social networking application which makes it compatible for visually challenged and deaf and dumb users:

\section{A. Screen Reader based Login/Sign-Up}

Normal User-Name and Password based authentication can be used by the deaf and dumb people, however, the visually challenged may need additional guidance from others to locate the User-Id and Password text boxes on the screen. Security and Privacy is also threatened by involving external helping agents, hence, a screen reader can be embedded into the application base which gives a voice output of the components and text on the screen at which the cursor points to. Many such screen reader softwares already exists in the market, one such software called NVDA will be utilised in the pipeline to create a demo prototype of the platform proposed[11].

\section{B. Video Transcription using Speech to Text}

This feature is proposed and implemented to mainly cater to the deaf and dumb people who cannot perceive the audio from any video posts uploaded on social netwroking platforms. Videos uploaded from well-known sources like YouTube have subtitles associated with them, however, any custom vidoes shot and uploaded by normal social media users will not have this feature. Therefore, there is a need to transcribe the audio to text format. The main idea is to extract the audio from the video being played, convert the audio to text using AI based speech to text recognition technology and display the same on the user interface[15].

\section{Video Transcription using Speech to Indian Sign Language(ISL)}

This feature is proposed and implemented to mainly cater to the deaf and dumb people who are not well versed with English Language, and more comfortable with Sign Languages. The use case is the same as the previous feature with one extra addition-output format will be sign language display instead of mere text. This can be implemented with a naive approach of mapping each character/word in the audio to text output with a predefined set of hand gestures. The same can be displayed on the user interface alongside with the transcripted text so that the user can use either of the features they are comfortable with. As a proof of concept, the demo prototype of the platform uses Indian Sign language gestures to show the transcribed text from the video. The same can be extended to other sign languages as well[7][10].

\section{Image Captioning using LSTM and OCR}

The main pillar of Social networking platforms in the present era is the visual content uploaded by its users. The visual content can be in the form of Images, GIFs, Memes and Image like posts with text. The normal screen readers available in the market like NVDA are not capable of explaining these visual contents to its users, which makes the visually challenged users unable to perceive the content. Hence, an attempt is made to solve this problem by implementing OCR and LSTM based Image captioning feature. The idea is here to pass the Image post via an OCR engine to recognise any text present in 
the Image, followed by an Image Captioning LSTM model which takes an Image as the input and returns a short description of the Image as the output, that can be converted to audio/speech using python libraries and sent to the User Interface enabling the visually challenged to listen to the image description.

\section{E. GIF Captioning using LSTM and OCR}

The previous Image captioning feature can be extended to the Graphics Interchange Format(GIFs) as well. GIFs can be considered as a series of Images stitched together explaining a small situation or a gesture. Hence, GIFs can be spliced into several frames and these frames can be sent to the OCR and LSTM Image captioning models for generating descriptions. The union of descriptions from all the frames can be finalised as the description of the GIF, which can be converted into audio and sent to the UI enabling the visually challenged to listen to the GIF descriptions.

\section{EXPERIMENTAL ANALYSIS}

\section{A. Speech To Text conversion}

Google's speech recognition software provided the python libraries for Speech to text conversion by taking an audio $\mathrm{mp} 4 /$ wav file as an input and providing the textual transcription of the same in English as the output. There are various speech recognition algorithms proposed by other companies like IBM Watson, Twilio, Amazon Transcribe, Microsoft Azure etc. Word Error Rate is a common metric used for comparing Audio Speech Recognition:

$$
\text { Word Error Rate }=(S+I+D) / N
$$

where, $\mathrm{S}$ is the number of substitutions, $\mathrm{D}$ is the number of deletions, $\mathrm{I}$ is the number of insertions and $\mathrm{N}$ is the number of words in the reference. The below graph represents the WER of various speech recognition APIs. Google's Speech recognition API and Microsoft's Azure have shown promising accuracies in recent times as seen.
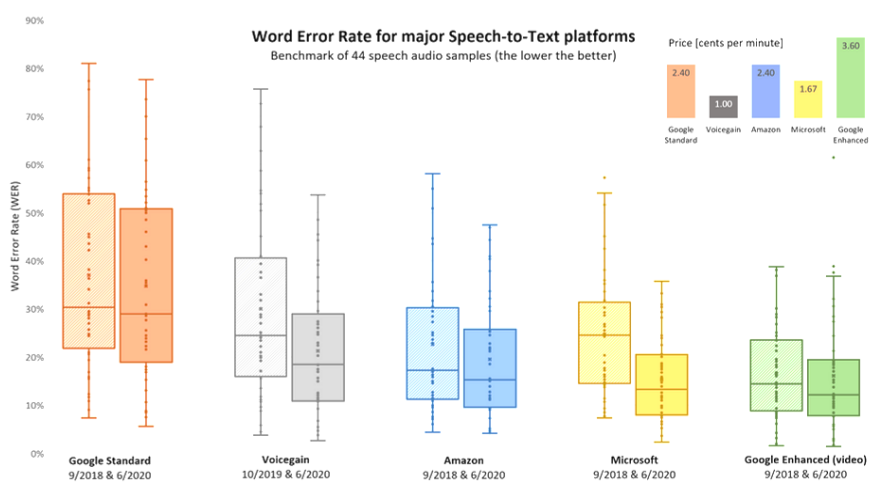

FIG 2. Word error rate for major Speech to text platforms.

\section{B. Text to ISL conversion}

The output from speech to text, is preprocessed using natural language processing and dictionary based machine translation is performed to obtain the required Indian Sign Language. In preprocessing, all the letters are converted to lowercase letters for further manipulation. In case the words after preprocessing exist in the predefined dictionary, then the corresponding GIFs of that phrase is displayed. The predefined dictionary has commonly used words like india,hello,good morning,saturday etc. If the word doesn't exist in the dictionary, then the letters of the word/phrase are counted and the visual of each letter will be displayed with a little delay.

In offline mode, using Sphix and Google Speech API , the ISL can still be displayed.

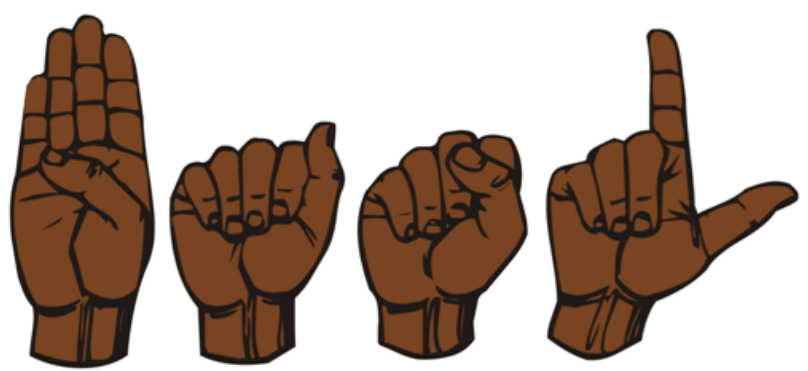

FIG 3. Indian Sign Language Gestures

\section{Image Captioning}

Image captioning blends the working of CNN and LSTM to generate an estimated description of the image provided as an input to the model. The model has three parts, firstly a pre-trained feature extractor is used to encode the images given as input to the model. Secondly, Keras embedding layer was used to generate word embeddings on the captions which were encoded earlier. Lastly, the embeddings were then passed into LSTM in which the image and text features were combined and sent to a decoder network to generate the next word.

The Bilingual Evaluation Understudy Score, or BLEU for short, is a metric for evaluating a generated sentence to a reference sentence. A perfect match results in a score of 1.0, whereas a perfect mismatch results in a score of 0.0 . For this model, Greedy Search and Beam Search both were used to generate the captions. Average Bleu Score was used to evaluate the captions generated for the test dataset.

TABLE I

Average Bleu Scores

\begin{tabular}{|c|c|}
\hline Greedy Search & 0.4623 \\
\hline Beam Search $(\mathrm{K}=3)$ & 0.4836 \\
\hline
\end{tabular}

\section{Optical Character Recognition(OCR)}

Tesseract[8] was originally developed at Hewlett-Packard Laboratories Bristol and at Hewlett-Packard Co, Greeley Colorado between 1985 and 1994. In 2005 Tesseract was open sourced by HP. From 2006 until November 2018 it was developed by Google. The latest version - Tesseract 4 adds a new neural net (LSTM) based OCR engine which is focused on line recognition, but also still supports the legacy Tesseract OCR engine of Tesseract 3 which works by recognizing character patterns. The accuracy and performance of Tesseract 4.0 LSTM based model when compared to its predecessors are shown in the below table. Tesseract 4.0 has more promising results, hence it was used to carry out the OCR task in the backend of a social networking application. 
TABLE II

Big Test at Google Data Center

\begin{tabular}{|l|l|l|l|l|}
\hline Engine & $\begin{array}{c}\text { Total } \\
\text { Char } \\
\text { Errors }\end{array}$ & $\begin{array}{c}\text { Word } \\
\text { recall } \\
\text { Errors }\end{array}$ & $\begin{array}{c}\text { Word Precision } \\
\text { Errors }\end{array}$ & $\begin{array}{c}\text { CPU } \\
\text { Time }\end{array}$ \\
\hline Tess 2.0 & 15.1 & 29.5 & 30.7 & 3.1 \\
\hline Tess 3.0 & 13.9 & 30 & 31.2 & 2.8 \\
\hline Tess 4.0 & 7.6 & 20.9 & 20.8 & 2.5 \\
\hline
\end{tabular}

\section{Sample ProtoType Using React JS and Flask}

The web application is based on various models which includes frontend developed using ReactJs and backend using Flask. React is a JavaScript library created by Facebook, which provides various frameworks to easily develop and use User interfaces. It's one of the leading languages for front-end development.Flask is used to define various endpoints in Python each of which is responsible for a particular service.These services are further invoked by the User interface.

In the backend, each model is a service and each service has a different endpoint. If the post is an image, the image is sent to the image captioning service. Here, first the OCR is used to check if there is any text in the image. If there is text, the text is converted to speech and if there is no text it is sent to the image captioning machine learning model to give the brief description of the posted image. If the post is a video, the audio of this video is converted to text and also ISL will be displayed based on the text. Now if the post is a GIF, the frames of this GIF are sent to the image captioning model where a brief description of each frame is obtained. All these descriptions put together gives a gist of the GIF. If the post is just text then, this text is converted to speech.

In the frontend, there is a screen reader which helps the blind to locate various buttons. For the deaf and dumb, the buttons are big in size and clearly visible with meaningful button names. On clicking each of these buttons the respective endpoints are called and the descriptions, text is visible below the post. The speech is clearly audible on clicking the respective button. For images, there are two buttons: text caption and image caption. The text caption button is enabled only when there is any text in the post or in the image and the image caption button is enabled only when there is an image without any text in it. For videos, there is a single button: start transcript which shows the text and immediately starts the ISL in a popup window.

For GIFs, there is a GIF transcript button which when clicked gives a description of the GIF. The text caption button will be enabled for videos and GIFs as well, if there is any text in the post.There are various features to change the theme of the website to dark or bright mode. Language can also be changed, here in the prototype spanish and english are the two languages available. There is a profile page as well, where the biodata, profile picture background picture can be edited. The posts of that particular person along with comments, likes can also be seen here.
The posts can be deleted as well. Since notifications are an important part of keeping people engaged, this feature is also implemented. Whenever a friend accepts a friend request, likes a post or comments on it, the person will instantly be notified.

Hence, just by clicking respective buttons and with the help of screen reader all the functionalities can be used and the specially challenged can overcome their limitations.

\section{CONCLUSION}

This paper has successfully proposed and implemented a prototype of a Social Networking Application which has extra features built, to make it more accessible for the visually impaired, deaf and dumb people. The screen reader based authentication, Image Captioning and GIF Captioning helps the visually impaired section to enjoy the visual content of social networking. The Video to text and Video to ISL translation feature enables the deaf and dumb people to enjoy the video content in social networking platforms. The underlying ML models have been trained and tested on several datasets and are the state of the art models in their respective domains. This social media application will be highly useful in pandemic situations to easily communicate the correct information[14].

The current trend in social networking platforms is the presence of memes, Memes can be relating to various domains, it can have text or image or both, and it mostly contains a sense of ironical meaning in them. Constructing a ML model to perceive this irony from memes will be a challenging task. Another challenge would be to summarise videos instead of providing mere transcripts. Extending Image captioning to videos is a computationally expensive and time consuming task, hence, accomplishing a better solution to this limitation is necessary.

\section{REFERENCES}

[1] Use of Social Media by People with Visual Impairments: Usage Levels, Attitudes and Barriers by Kristin Skeide Fuglerud Ingvar TjøstheimBirkir Rúnar Gunnarsson Morten Tollefsen,Computers Helping People with Special Needs, 2012

[2] Visually impaired users on an online social network by Shaomei Wu and Lada Adamic,Proceedings of the 32nd Annual ACM conference on Human factors in Computing Systems,2014.

[3] A Survey on the Use of Mobile Applications for People Who Are Visually Impaired by Nora Griffin-Shirley, Devender R. Banda, Paul M. Ajuwon,Jongpil Cheon, Jaehoon Lee, Hye Ran Park, and Sanpalei N. Lyngdoh,Journal of Visual Impairment \& Blindness, July-August 2017

[4] Social and Communication Apps for the Deaf and Hearing Impaired by Mrim Alnfiai,Srini Sampali,Maraim Alnefaie,2017,International conference on computer and application.

[5] Impact of social media on the way disabled people integrate to the modern society by Masuma H. Mammadova, Sanan M. Ahmadov,Problems of information society, 2017

[6] A Systematic Literature Review of the Application of Information Communication Technology for Visually Impaired People,Mahfuz Ashraf,Najmul Hasan,Lundy Lewis,Md Rashadul Hasan,Pradeep Ray,The International Journal of Disability Management Research ,2017

[7] Sign Language Recognition for Deaf and Dumb People Using Android Environment by A. Gayathri ,A. Sasi Kumar, International journal of current engineering and scientific research(IJCESR),2017. 
[8] An Overview of the Tesseract OCR Engine Ray Smith Google Inc. theraysmith@gmail.com

[9] Accessible social media and apps give a voice to people with disabilities by Narmeen Shigri,medium article,2017

[10] The New Agora: Social Media as a Vector for Sign Language as a Language of Culture, Identity and Inclusion of the Deaf by Cayley Guimarães,Sueli Fenandes ,Proceedings of the 51st Hawaii International Conference on System Sciences, 2018

[11] Blindness and Social Media in India by Soumya Thankam Varghese, Dr.Maya Rathnasabapathy ,International Journal of Engineering \& Technology,2018

[12] Improving Social Inclusion for People with Physical Disabilities: The Roles of Mobile Social Networking Applications (MSNA) by Disability Support Organizations in China by Hyeon-Cheol Kim and Zong-Yi Zhu,Int J Environ Res Public Health. 2020

[13] Sign Language Generation System Based on Indian Sign Language Grammar by Sugandhi, Parteek Kumar,Sanmeet Kaur ,ACM Transactions on Asian and Low-Resource Language Information Processing, 2020

[14] Science communication for the Deaf in the pandemic period: absences and pursuit of information by Alexandre G. Silva, Tiago Batista, Felipe Giraud, Andrea Giraud, Flavio Eduardo Pinto-Silva, Julia Barral, Juan Nascimento Guimarães and Vívian rumjanek, Journal of Science Communication(JCOM),2020

[15] Analysis of emerging technologies for the social inclusion of people with hearing disabilities: a review of the scientific literature from 2005 2020 by Henry Melgarejo-Nagata,Michael Cabanillas-Carbonell,European Journal of Molecular \& Clinical Medicine,2021 